\title{
Pedro Schwartz*
}

\section{EL TEOREMA DE COASE PUESTO A PRUEBA EN CHINA}

Generalmente se presenta la doctrina de Coase como un teorema estático que busca explicar la corrección espontánea de defectos del mercado por acuerdos de los afectados. Sin embargo, según el teorema como lo formuló Stigler, esos resultados solo se alcanzan bajo los cuestionables supuestos de que los costes de transacción son nulos, los derechos de propiedad están bien definidos y la distribución del beneficio de los acuerdos no afecta al resultado. Lo irreal de esas condiciones hace que, por desgracia, siga aplicándose la teoría estatista de los defectos del mercado cual la promulgó Pigou. Pero el espectacular progreso de la economía china después de Mao, tal y como la analizan Ronald Coase y Ning Wang (2012), parece haber ocurrido sin ajustarse en nada a lo expresado en ese teorema.

\section{The Coase Theorem tested in China}

The Coase doctrine is usually presented as a static theorem purporting to explain the spontaneous correction of market defects by agreement between affected parties. However, as Stigler formulated it, those results are only reached under the questionable assumptions of zero transaction costs, well-defined property rights, and independence from the distributive effects of the agreements. The lack of realism of these assumptions has resulted in the unfortunate persistence in applying the statist theory of market defects as proclaimed by Pigou. The spectacular progress of the Chinese economy after Mao, as analyzed by Ronald Coase and Ning Wang (2012) seems to have happened without following the conditions of that theorem.

Palabras clave: Teorema de Coase, derechos de propiedad, costes de transacción, efectos distributivos, experiencia económica china, impuestos pigovianos.

Keywords: Coase Theorem, property rights, transaction costs, distributive effects, China's economic experience, Pigovian taxes.

JEL: D23, K11.

\footnotetext{
* Universidad Camilo José Cela, Madrid.

Agradezco la ayuda del Profesor Cabrillo durante la redacción de este trabajo; y especialmente las muy críticas y acertadas reflexiones del referee anónimo. Leí una primera versión en la reunión de la Mont Pelerin Society en Hong Kong en septiembre de 2014.

Versión de julio de 2020.

DOI: https://doi.org/10.32796/ice.2020.915.7080
} 


\section{Introducción: el de Coase, un éxito aparente}

El intento de Ronald Coase de arrumbar de una vez para siempre la teoría del bienestar de Arthur Pigou no ha tenido éxito. El propio Coase lo reconoció. Al comentar un cuarto de siglo más tarde su famoso artículo «The Problem of Social Cost» (1960), Coase lamenta «la extraordinaria influencia que el enfoque de Pigou sigue teniendo entre los economistas modernos» (Coase, 1988, p. 159). Nuestra profesión recita de carrerilla el llamado Teorema de Coase, pero ni lo entiende ni por tanto lo aplica. Hoy en día, en cuanto un economista se enfrenta con algún fallo de mercado en forma de bienes públicos o de efectos externos, es a Pigou a quien acude: inmediatamente pide que la Autoridad cargue un impuesto o conceda una subvención para corregir ese defecto.

La razón por la que se deja de lado la doctrina de Coase es que el teorema por la que se la conoce no hace justicia a su verdadera complejidad teórica. La cantinela es conocida: «siempre que los derechos de propiedad estén bien definidos y los costes de transacción sean nulos, los acuerdos entre las partes podrán resolver las externalidades, si no tomamos en cuenta la redistribución de rentas consiguientes a tales acuerdos». Esa formulación es prácticamente una tautología, pues el teorema está definido de tal manera que no tiene más remedio que ser cierto. Un defecto de mercado se resolverá por sí solo aún sin que se den las tres condiciones del teorema de Coase, porque estas sobran si, como veremos a lo que se indica Cooter, la situación es de competencia perfecta (Coase, 1988, p. 158). Si hay perfecta información, perfecta divisibilidad de factores y perfecta discriminación de bienes, el problema de los efectos externos se esfuma. Mi tesis es que la formulación del teorema de Coase de los libros de texto no es más que una parodia del pensamiento de Coase y lo deja expuesto a críticas como la de Robert Cooter en «The Cost of Coase» (Cooter, 1982) a la que acabo de aludir; o la de James Buchanan «The Irrelevance of Transaction
Costs» (Buchanan, 1984). Lo irreal del supuesto de perfecta competencia y costes de transacción nulos del teorema de Coase explica por qué la economía del bienestar de Pigou sigue viva.

Muy al contrario, Coase quiso llamar la atención sobre lo complejos que son los casos reales de solución espontánea de externalidades, como el del progreso de China hacia el capitalismo. En casos como ese, los derechos de propiedad no vienen dados, sino que se modifican durante las negociaciones. Además, lejos de ser nulos, hay costes de transacción que hay que superar para alcanzar acuerdos; estos costes de transacción son de al menos tres tipos: $i$ ) carencias de información y comunicación; ii) costes nacidos de la conducta de los free-riders; y iii) comportamiento estratégico de las partes para apropiarse de los beneficios del cambio, hasta el punto de que alguno de los implicados no quiera moverse hacia un óptimo de Pareto por considerar inaceptable la distribución de los beneficios. Es Cooter el que ha hecho notar que muchas de estas complicaciones desaparecen cuando se razona como si la competencia fuera perfecta.

No es por ese camino por el que hay que transitar. Dice Coase (1988, cap. 6, p. 174): «Un mundo sin costes de transacción se ha descrito a menudo como un mundo a la Coase. Nada puede ser menos cierto». La reducción de la doctrina de Coase al teorema de Stigler esconde el fenómeno que verdaderamente interesa a Coase: la espontánea aparición de instituciones y reglas que ayudan a resolver situaciones subóptimas.

Coase mereció el galardón del Nobel de economía, no por ese «teorema» que él nunca formuló ${ }^{\text {, }}$ sino por haber fundado la teoría de la organización industrial (Coase, 1988, cap. 3); y, más generalmente, por haber abierto el camino a la nueva economía institucional (Coase, 1988, cap. 6) - o teoría de las repercusiones legales y económicas de la existencia de costes de transacción. Coase, aparte

\footnotetext{
1 Véase lo que dice Coase sobre su teorema (Coase, 1988, pp. 157-158).
} 
de su ensayo sobre el coste social (Coase, 1960), estudió esas cuestiones en otros trabajos en los que tampoco mencionó el dichoso teorema: en especial, aquel en el que explicó por qué aparecen empresas en el mercado económico (Coase, 1937), o por qué es conveniente subastar los espacios del espectro radioeléctrico (Coase, 1959), o cómo es que hubo inversores privados en la construcción de faros marítimos cuando su principal producto es un bien público (Coase, 1974). Pero aquí no me ocuparé de los efectos institucionales de los costes de transacción, sino de un caso de esterilidad del «teorema de Coase»: lo inútil que es ese teorema para la búsqueda de alguna explicación racional del notable desarrollo económico de China después de Mao.

La evolución de la economía china hacia situaciones Pareto-óptimas de los sistemas capitalistas es un contraejemplo del teorema de Coase: en principio lo refuta ${ }^{2}$ y por tanto pide una reformulación. En la China posterior a Mao no se daban en absoluto las condiciones que, según ese teorema, habrían explicado la extraordinaria y exitosa transformación que ha asombrado al mundo. Los derechos de propiedad se iban delimitando solo a medida que la economía se movía hacia el capitalismo. Los costes de transacción no eran ni mucho menos nulos, pues la información y su comunicación tenían que superar enormes obstáculos políticos. La existencia de imitadores o freeriders, en vez de dificultar las transacciones, las generalizaba por un efecto de imitación. Los beneficios de medidas Pareto-óptimas, lejos de dificultar los acuerdos, a menudo ayudaban a financiar los sobornos que permitían esquivar reglamentaciones caprichosas o contraproducentes.

En eso radica el interés del libro de Ronald Coase y Ning Wang (2012) How China Became Capitalist. Los autores señalaron que en la China post Mao el Estado, queriendo o sin querer, iba modificando continuamente

\footnotetext{
2 En la figura lógica del modus tollens, un solo contra-ejemplo basta en principio para concluir la falsedad de una proposición universal. Popper (1958, cap. I, secc. 6, p. 41).
}

los derechos de propiedad bajo la presión emprendedora de los individuos, de las empresas, y de las autoridades locales. Se alcanzaban acuerdos y se ejecutaban contratos en circunstancias legalmente muy poco propicias. Y la prosperidad resultante fue tan grande que los conflictos distributivos, aunque existentes, se redujeron al mínimo, pues había grandes ventajas para todos.

Antes de presentar el contraejemplo de la teoría del bienestar pigoviana, a saber, la evolución hacia el capitalismo de la economía china traído por Coase y Ning, recordaré a mis lectores cómo nació el teorema de Coase.

\section{Stigler y el «teorema de Coase»}

George Stigler en sus Memorias (1988) cuenta la historia de la famosa cena de 1960 en la casa del cuñado de Milton Friedman, Aaron Director, para discutir «cómo era posible que un economista tan bueno como Coase pudiera cometer un error tan evidente» como el de criticar la teoría de Pigou (Stigler, 1988, p. 75). Veinte economistas, entre los que se encontraban Friedman y el propio Stigler escucharon a Coase presentar la proposición herética de que la forma en que se utilizan los recursos económicos no depende de a quién se le adjudica la responsabilidad legal por daños y perjuicios o a quienes se asignan los derechos legales de propiedad (Stigler, 1988, p. 76) ${ }^{3}$.

Tras intervenir Coase, la minoría de uno a su favor al comienzo de la cena se transformó a la unanimidad de veintiuno al final.

El primer paso en el camino de las propuestas de intervención pública para corregir los defectos del mercado lo había dado J. S. Mill en su capítulo sobre «Los límites del laissez faire» de sus Principios de economía

\footnotetext{
3 Como dice Cooter (1982, p. 15), una interpretación correcta del teorema de Coase no implica que el acuerdo final entre las partes sea independiente de la adjudicación de las responsabilidades legales o de los derechos de propiedad, sino que, cualquiera sea esa asignación final de los recursos, el resultado será eficiente.
} 
política (Mill, 1848, 1965, libro iv, cap. xi). Luego, la profesión económica fue ampliando el número de casos en los que un mercado libre no produce resultados eficientes ${ }^{4}$. Principalmente fue Pigou, en su libro sobre $L a$ Economía del Bienestar (1920), quien llegó a la conclusión de que el bienestar social no coincidía normalmente con el bienestar privado que buscan los agentes del mercado: adujo un sinnúmero de efectos externos por los que los mercados resultaban ser ineficientes. En el siglo XX, esta idea de Pigou se convirtió en la doctrina aceptada por la profesión. Hoy el argumento se ha hecho casi proverbial con la repetida cita en las clases de microeconomía de ejemplos como la fábrica que emite humos o el descubrimiento de la penicilina por el Doctor Fleming. Se suele concluir que en esos casos es lícito que el Estado grave con impuestos o conceda subvenciones a la actividad privada para conseguir el máximo bienestar social.

En esa cena, Ronald Coase, para mostrar que las propuestas de Pigou no eran de validez universal, utilizó el ejemplo extremo de una situación en la que los costes de transacción eran nulos. Además, y pese a ser un modelo muy genérico, pudo deducir algunos principios que iban muy en contra de la extendida doctrina de los defectos del mercado5: que eran concebibles mercados con defectos óptimos; que era posible que las partes implicadas corrigieran externalidades de mutuo acuerdo; que los costes de operar en el mercado se obviaban a menudo internalizándolos al unir ambas partes en una empresa ${ }^{6}$; que el óptimo resultante de una negociación privada podía dar lugar a una distribución de los derechos de propiedad diferente de la inicial; y que las decisiones de los tribunales sobre quién tiene derecho a contaminar o a ser indemnizado; y las reglas dictadas por las autoridades, como la de que «el

\footnotetext{
4 Schwartz (1972, cap. 6). Como podrá ver el curioso lector, mi postura elogiosa de la teoría de los límites del laissez faire expuesta por Mill ha cambiado sustancialmente.

5 Véase cómo explica esos principios Coase (1988, vi, pp. 157-185).

623 años antes, Coase había publicado su ensayo «The Nature of the Firm» (Coase, 1937), en el que explicaba que el coste de utilizar el mecanismo del mercado explicaba la aparición de la empresa.
}

que contamina, paga»: podrían resultar el mayor daño para todos.

La mera posibilidad de estas conclusiones era (y es) chocante para los numerosos discípulos de Pigou.

Stigler recogió la idea de Coase en un teorema que la reducía a un modelo simplista. En su recuerdo de la cena, dijo que Coase les había pedido que supusieran costes de transacción nulos. Si Coase partió del supuesto de costes de transacción nulos en esa cena debió de ser porque sus amigos de Chicago tendían a razonar de esa forma abstracta, aunque para la mayoría de los casos los costes de transacción no son nulos. El supuesto era, pues, «heroico», que dicen los angloparlantes, pero era este un modo de razonar al que los economistas estamos muy acostumbrados: una vez aceptado el supuesto, es posible demostrar el caso extremo, que luego, debidamente modificado sirve para explica casos reales, siempre más complicados. Pues bien, en este caso el supuesto de costes de transacción nulos lleva a lo que en lógica se llama una «falacia de división», en la que uno cae cuando supone que las partes de un conjunto tienen las mismas propiedades que el conjunto.

Para dar un ejemplo de costes de transacción nulos, Stigler eligió el caso extremo de que tanto el que contamina (el propietario del ganado) como la parte dañada (el cultivador de maíz en un campo abierto) se fusionaran en una sola empresa. En ese caso el propietario único organizaría la producción sin costes de transacción, para así maximizar el producto conjunto de ambas actividades ${ }^{7}$. Eso es lo que Stigler llamó «el teorema de Coase» (Stigler, 1988, p. 77).

\section{Cooter sobre el teorema de Coase}

Una de las críticas más duras dirigidas a Coase en materia de coste social es la de Robert Cooter en el

\footnotetext{
7 El caso de un único dueño de la granja productora de maíz y de vacuno también está expuesta en Stigler (1966, p. 113). Coase lo había explicado en más detalle en (Coase, 1937); véase la nota 2, más arriba.
} 
artículo que lleva el ingenioso título de «The Cost of Coase» (Cooter, 1982). Señala Cooter primeramente que en los procesos de negociación hay elementos erróneamente clasificados bajo la rúbrica de costes de transacción. "Las negociaciones tienen resultados no cooperativos incluso cuando el proceso de negociación no tiene coste» ${ }^{8}$. Más demoledor es que muestra que el teorema de Coase es válido solo en condiciones de competencia perfecta. La razón por la que esto es así es que los efectos redistributivos de hacer más eficientes los mercados generan disputas sobre el reparto de las ganancias y, por tanto, indeterminación en las negociaciones. Un mercado plenamente competitivo es un juego de cooperación puro, donde la distribución de las ganancias derivadas del avance hacia un óptimo de Pareto tiene un efecto insignificante o nulo sobre el resultado, ya que los partícipes no pueden amenazarse mutuamente con movimientos estratégicos. Cuando los concernidos son pocos, siempre existe el peligro de que el intento de una parte de acaparar la mayor parte del beneficio de la acción dé lugar a una ruptura de las negociaciones y a pérdidas generalizadas.

Medema (1995) añade a esto que, en términos generales, habrá dos salidas de las actitudes mutuamente destructivas: los coasianos propondrán que se eliminen las barreras legales o administrativas que dificultan las transacciones, para que la negociación se lleve a cabo en una situación tan plenamente competitiva como sea posible; los hobbesianos por el contrario querrán crear un Leviatán con poderes suficientes para contener la violencia de los partícipes y forzar una solución «óptima» ${ }^{9}$.

La conclusión de la crítica de Cooter es que el teorema de Coase es de aplicabilidad limitada por ser válido solamente en mercados perfectamente

\footnotetext{
8 En la página 28 llega a la conclusión antiintuitiva de que «es más fácil tener un comportamiento estratégico cuando la comunicación es barata» y la negociación puede prolongarse sin fin (Cooter, 1882, pp. 17-18).

9 Como se verá, en el caso de China no fue necesaria la aplicación de ninguna de estas dos soluciones: bastó con la corrupción.
}

competitivos. ¿Hemos de contentarnos con esta conclusión tan mínima?

La realidad social de la China capitalista me ha llevado, primero, a quitar importancia a la crítica de Cooter de que el análisis de Coase no pasa de ser solo aplicable a situaciones de competencia perfecta. De hecho, las tres condiciones de nulos costes de transacción, definición de derechos de propiedad, y olvido de efectos redistributivos sobran si se supone que el marco es de libre (no perfecta) competencia. En segundo lugar, intentaré ver con lentes de Buchanan la notable reforma institucional ocurrida en China durante esos años.

\section{La perspectiva institucional de Buchanan}

Buchanan llega aún más lejos cuando retoma algunas de las observaciones de Cooter sobre el carácter complejo de esos costes (Buchanan, 1984, pp. 267-273). Estos no solo incluyen prohibiciones y comisiones a menudo impuestas por las autoridades; también incluyen restricciones de información y comunicación; $y$, también la conducta de freeriders y el comportamiento estratégico.

Pero vayamos al fondo de la crítica de Buchanan. Sostiene, en ese trabajo, subtitulado nada menos que "The irrelevance of transaction costs» (1984), que Coase y muchos coasianos parecen pensar que en cualquier situación existe una única asignación eficiente de recursos que un observador externo podría percibir (Buchanan, 1984, p. 262). Propuso Buchanan que el análisis de Ronald Coase se reinterpretara partiendo de un punto de vista «subjetivistacontractualista» o también «Austriaco-Wickselliana».

Empezó, como era su costumbre, negando que los óptimos fueran observables por terceros. La falacia en las formulaciones habituales del teorema de Coase reside en considerar que el óptimo se puede definir de manera objetiva. Es un error decir que una situación sufre externalidades porque no se haya llegado a un punto óptimo objetivamente observable. «Es lamentable [dijo] que Coase presentara su argumento [...] 
en gran medida en términos de comparaciones de daños y beneficios supuestamente medibles de forma objetiva y determinados de forma independiente». Es un error, prosigue, pensar que existe una asignación «eficiente» [...] y que cualquier observador externo puede determinarla conceptualmente. Un observador externo no puede determinar si un trato ha alcanzado un nivel óptimo. Cada negociador percibe lo que es óptimo para él o ella y en consecuencia solo él ve si llegó o no pudo llegar a un intercambio satisfactorio. Las operaciones realizadas, sean o no en condiciones de competencia perfecta, serán siempre "óptimas», aunque el marco institucional no lo sea. Que los costes de transacción sean altos o bajos no importa a la hora de llevar a cabo el trato: este será óptimo «dado el entorno institucional» (Buchanan, 1984, p. 264, cursivas en el original).

Esto parece sacado del Candide de Voltaire (1779), en el que todo es para bien en el mejor de los mundos posibles, ya sean las repetidas violaciones de Cunegunda, la amada de Cándido, o el terrible terremoto de Lisboa, del año 1755. No tal, sugiere Buchanan. Cuando las partes en una transacción descubren que no están satisfechas con el acuerdo alcanzado tratarán de cambiar el marco institucional en el que operan, siempre y cuando puedan negociar sin interferencias políticas. «Si las restricciones [institucionales] iniciales abocan a la «ineficiencia», los potenciales transactores encontrarán ventajoso invertir recursos en hacer esfuerzos para eliminarlas» (Buchanan, 1984, p. 268).

El acuerdo para cambiar las reglas bajo las que realizar los intercambios sería una señal de que los resultados alcanzados [...] en el marco de normas previamente existente son menos preferidos o valorados que los patrones que se espera se generarán en el marco de las reglas una vez cambiadas (Buchanan, 1984, p. 265).

De su análisis extrae Buchanan dos consecuencias. La primera es que la noción de costes de transacción es incompleta (y se supone necesita concreción), como había dicho Cooter. Estos «costes de transacción observados objetivamente» son un cajón de sastre de deficiencias de información, prohibiciones de las autoridades, ocultación voluntaria de las intenciones, comportamiento estratégico, o freeriding antisocial, pero no instrumentos útiles de análisis. La segunda es que los llamados «costes de transacción» [o] barreras a la «eficiencia» en la asignación de recursos se pueden analizar de manera más apropiada en el contexto de hipótesis de reforma institucional (Buchanan, 1984, p. 275).

Ahora bien, el desplazamiento de la búsqueda de la «eficiencia» al plano superior de la reforma institucional debe tratarse de nuevo como una cuestión subjetiva, donde determinados grupos de personas pueden intentar cambiar las reglas que no les gustan.

Buchanan explica lo subjetivo de la noción de «eficiencia» con una reinterpretación del proverbial «dilema del preso». El resultado subóptimo al que dice la generalidad de los autores que cuando llegan los dos delincuentes detenidos en régimen de incomunicación, es que confesarán el crimen porque no pueden confiar en el silencio del otro. Subraya Buchanan que esta conclusión es ineficiente para los delincuentes, pero quizás no lo sea para el resto de la sociedad. Una solución de la «ineficiencia» percibida por los delincuentes puede resolverla la fraternidad de los mafiosos imponiendo la ley del silencio bajo la amenaza de represalias. Sin embargo, es posible que para los ciudadanos amantes de la legalidad sea mejor que los delincuentes delaten a sus cómplices. Los diferentes grupos sociales pueden tener diferentes visiones de "lo que es eficiente» y lo buscarán a su manera. Veremos que esta forma subjetiva y diferente de entender la eficiencia arroja alguna luz sobre el avance de China hacia el capitalismo.

La búsqueda de un cambio del marco institucional como remedio para las externalidades parece una utopía, porque tendemos a leerlo como propuesta de cambios de la Constitución política del país, que es de lo que Buchanan se ocupó mayormente. Como es sabido, Buchanan distinguía en la ordenación de una sociedad, la Constitución, siempre 
adoptada por unanimidad, y su aplicación día a día, objeto del combate político. Aquí, sin embargo, se refiere a reformas institucionales más pequeñas, cual son las tratadas por Coase en su trabajo sobre la naturaleza de la empresa (Coase, 1937). Insatisfechos con la solución de los efectos externos por acuerdo o por sentencia judicial, las partes deciden crear conjuntamente una empresa. Así resuelven sus diferencias el ganadero y el agricultor en el ejemplo de Stigler que hemos visto más arriba. Y esa formación de empresas, cooperativas, y asociaciones entra dentro del conjunto de las decisiones sociales unánimes que Buchanan considera legítimas, aunque ello pueda sorprender al amable lector. En efecto, esos acuerdos cooperativos son unánimes en el sentido de que las partes implicadas lo quieren y el resto de la sociedad se muestra indiferente. Es una unanimidad nemine discrepante, como de hecho lo son todas las situaciones de óptimo paretiano $^{10}$.

\section{Coase sobre la China capitalista}

Vayamos al experimentum crucis. Inesperadamente y hacia el final de su larga vida Ronald Coase comenzó a interesarse por la evolución de China hacia el capitalismo. El resultado de este nuevo interés es el referido libro que escribió con Wang Ning, titulado How China Became Capitalist (2012) que publicó a sus 102 años. Vista desde el exterior, la transformación de China es asombrosa; desde el interior, según la relata este libro, es nada menos que un milagro.

La principal aportación de este libro, comparada con la de otros menos perspicaces de la transformación económica de China, es que el cambio no fue el resultado de una cuidadosa planificación del Gobierno. La transformación ocurrió desde abajo, de forma descentralizada e imprevisible. La autoridad central supo abrir la puerta al cambio y adaptarse. Varias veces estuvo en peligro, como cuando todo se paralizó tras la masacre

\footnotetext{
10 Sen (1970, cap. 2)
}

de Tiananmen en 1989. Tampoco está el futuro libre de peligro, especialmente en vista de los crecientes obstáculos al avance hacia una mayor libertad personal y política. Es cierto que los autores se equivocaron al decir al final de su libro que la falta de un mercado de ideas, [en China], es directamente responsable de la falta de innovación en ciencia y tecnología, el talón de Aquiles en el crecimiento del sector manufacturero en China. (Coase y Wang 2012, p. 193).

Está claro que su visión de China era la de una economía principalmente fabril al servicio de otras economías más adelantadas. Las cosas han cambiado mucho desde principios de siglo. China va pareciéndose cada vez más a la Alemania imperial de antes de la Primera Guerra Mundial, admirada en el mundo entero por sus investigaciones científicas, su tecnología, sus fuerzas armadas... y su intervencionismo social. Esas aventuras imperialistas suelen tener mal fin. Sin embargo, temo que, a medida que el país se militariza y disciplina, se agoste la libertad que necesita el adelanto de los conocimientos.

No es esa la cuestión que me ocupa. Desde el punto de vista de este artículo, el interés del libro radica en su tesis de que la transformación de China de una tiranía socialista en una economía capitalista sui generis no la planeó el Partido Comunista ni lo hicieron los diferentes gobiernos que dirigieron el país después de Mao. Fue el resultado no intencionado de una política encaminada a la conservación del socialismo a través del crecimiento económico. Los líderes chinos, en especial Deng Xiaoping, estuvieron dispuestos a tolerar la actividad privada, siempre y cuando mantuvieran el control de las empresas estatales y los capitalistas no amenazaran el socialismo oficial. Ello permitió la expansión inesperada de lo que los autores llaman «las cuatro fuerzas marginales» - marginales en el sentido de desarrollarse al margen del mundo oficial: «la agricultura privada, las empresas privadas tanto rurales como urbanas, el emprendimiento individual, y las Regiones Económicas Especiales» (Coase y Wang, 2012, p. 164). Se permitió primero el cultivo privado de las tierras pertenecientes 
a las comunas y la venta libre de su producto. Luego se adscribió de facto la posesión de las tierras a las familias. En paralelo con las grandes empresas públicas, aparecieron sociedades anónimas que incluso pudieron cotizarse en las Bolsas de Shanghái y Hong Kong y se permitió el emprendimiento individual, sobre todo de nuevos productos y servicios. $Y$ notable fue la arriesgada apuesta de Deng por la creación de zonas de libertad económica alrededor de algunos puertos marítimos y fluviales.

\section{Una reforma venida de abajo}

Esas nuevas libertades se desarrollaron de manera inconsciente. No eran consecuencia de un plan de gobierno concertado para eliminar las barreras administrativas o reducir los costes de transacción. En esa sociedad comunista, los costes de transacción debían ser casi insuperables para los empresarios individuales, sobre todo al principio, cuando corrupción y empresa capitalista se castigaba prácticamente al igual. La liberalización del comercio mundial permitió que las exportaciones chinas confirmaran el acierto de la nueva política económica. Las soluciones de mercado fueron sobre todo fruto de acuerdos entre personas privadas. Se apelaba a las autoridades estatales y locales para evitar reglamentaciones y consolidar nuevos derechos de propiedad. Como subrayan los autores, China no delimitó primero los derechos de propiedad y especificó otras reglas institucionales relevantes para luego permitir que las fuerzas del mercado asignaran esos derechos al mejor postor. [...] Durante las dos primeras décadas de la reforma, mientras China seguía comprometida con el socialismo y se oponía a la privatización total de los activos del Estado, los derechos que obtenían los agentes privados del Estado eran objeto de negociación individual (Coase y Wang, 2012, p. 172).

El cambio se aceleró porque el Estado siguió los dictados de la empresa privada sin siquiera tiempo «para establecer derechos hasta que se determinaron sus valores económicos en régimen de competencia».
Como las condiciones económicas cambiaron al paso del tiempo [... el] Estado tuvo que revisar y redefinir con frecuencia la estructura de derechos de propiedad. [...] (Coase y Wang, 2012, p. 173).

La «corrupción» desempeñó un papel fundamental en el marco de la teoría de Coase. Las nuevas libertades económicas permitían la acumulación de grandes fortunas, pero esas libertades no habrían florecido sin la redistribución semitolerada de las ganancias capitalistas entre empresarios y autoridades.

\section{No hacía falta competencia perfecta}

De este modo, los beneficios de pasar de una economía socialista que funcionaba mal a una economía capitalista en cierto modo limitada, pero productiva, constituyeron el incentivo para consentir las reformas. No todos los incentivos eran pecuniarios: muchas autoridades provinciales y sobre todo Deng Xiaoping buscaban el beneficio político dimanante del progreso social. No había ninguna necesidad de un régimen de competencia perfecta para evitar los conflictos surgidos del reparto de la nueva riqueza. Tampoco tuvo que actuar el Leviatán para evitar los conflictos con motivo del reparto de los frutos de la mayor productividad. Hubo conflictos, pero estos se produjeron sobre todo por la inoportuna interferencia de aquellos elementos autoritarios de la dirigencia que temían el crecimiento capitalista.

La «corrupción» fue, pues, una mejor vía para redistribuir el producto de las mejoras que lo que habría suministrado la planificación y el control centralizado. La riqueza creció tanto que hubo espacio para una mejora de todos - excepto para los políticos que preferían el poder a la prosperidad. Apliquemos el dilema del preso a la Buchanan. Hemos visto que normalmente este modelo se presenta como un fracaso del libre mercado porque los dos presos, al perseguir su interés, se encuentran en una situación peor de la que desearían. Divide Buchanan la sociedad en dos grupos, los delincuentes y la gente honrada. El mantener 
a los delincuentes incomunicados puede ser contrario a los intereses de estos pero beneficioso para el resto de la sociedad. En China jhan ganado los delincuentes capitalistas! Los delincuentes se ponen de acuerdo y las fuerzas de la ley se adaptan. El Estado y sus gobernantes habrían preferido controlar e incluso impedir el movimiento de la minoría desobediente pero los nuevos empresarios y los cargos locales tomaron las riendas, a veces con gran peligro para ellos mismos. Así es como se entiende la eclosión del capitalismo en China comunista. La empresa privada urbana era la más expuesta a la acusación de «delito económico» pero siempre había individuos que burlaban lo policiaco de sistema.

Una viñeta dibujada, por así decir, por los autores explica lo ocurrido mejor que largas disquisiciones. Cuentan la singular historia de Nian Guangjiu en la provincia de Anhui (Coase y Wang, 2012, pp. 77-78). Analfabeto y sin empleo, Nian encontró una manera de tostar las semillas de sandía que las hicieron las preferidas por los viandantes de las calles de Wuhu. En 1980 la gente hacía cola para comprarlas y Nian comenzó a emplear a personas que no eran de su familia - un caso de explotación capitalista. La esposa de Nian temía que le encarcelaran, como había ocurrido bajo Mao. Pero llevaron el caso ante Deng Xiaoping, que, siempre pragmático, se inclinó por esperar y ver. Las exquisiteces de Nian empezaron a conocerse como «las semillas de sandía del chalado». Aun así, Deng tuvo que intervenir dos veces, en 1984 y 1992, para salvar a Nian de un arresto. La desobediencia sale victoriosa.

\section{Un punto de vista evolutivo}

Al introducir la evolución institucional espontánea y al abandonar la idea de costes de transacción nulos y la delimitación inicial de los derechos de propiedad, Coase abrió la puerta a una fructífera aplicación de su doctrina. Esta línea es la que luego han seguido otros dos galardonados con el premio Nobel de Economía,
Oliver Williamson y Elinor Ostrom: el primero, al estudiar el modo en que las instituciones del capitalismo, en especial las relaciones entre empresas, reducen los costes de transacción (Williamson, 1985); y los fallecidos Elinor Ostrom y su marido Vincent, al estudiar las innovaciones institucionales espontáneas o cooperativas que corrigen fallos en el uso de recursos naturales mejor que las administraciones públicas o el mercado (Ostrom, 2012). Los desarrollos teóricos de estos tres premios Nobel, Coase, Williamson y Ostrom, contribuyen a indicar que la continua denuncia de los profundos defectos del capitalismo quizá sea algo exagerada.

\section{Conclusión: efectos externos de los impuestos y subvenciones a la Pigou}

La comprensión de la doctrina de Coase, pues, tiene una importancia que va más allá de lo histórico. Cierto es que la doctrina de Coase exige mucha atención a los detalles de la realidad y alguna fe en que los individuos son capaces a veces de resolver los problemas planteados por externalidades negativas y positivas.

Coase no era un enemigo sistemático de la intervención pública mas para él esa intervención no puede ser formulista y unilateral. Como nota Coase en sus «Notas sobre el coste social» (Coase, 1990, pp. 179 ss.), los impuestos pigovianos cargados con el fin de corregir un efecto externo negativo pueden no incluir todo el daño social que indirectamente causan. Así, los habitantes vecinos de la fábrica a la que cobra una contribución para reducir la suciedad que causaba deberían pagar (al fabricante) un tanto por la mejora del aire que respiran. Otro ejemplo de costes pigovianos que no se computan es el de las medidas propuestas para reducir la cantidad de $\mathrm{CO}_{2}$ emitida por los humanos. Sí suelen incluirse cálculos estimativos del gasto directo previsto para alcanzar la meta de emisiones cero. Quedan, sin embargo, fuera del cálculo muchos de los costes de oportunidad. Así, se nos exhorta apocalípticamente a llevar una vida espartana, pero nadie dice nada sobre el 
«malestar» que nos causaría a muchos el tener que convertirnos en veganos.

El que Pigou siga siendo el referente para la corrección de los llamados defectos del mercado tiene consecuencias negativas.

\section{Referencias bibliográficas}

Buchanan, J. M. (1984). Rights, Efficiency, and Exchange: The Irrelevance of Transaction Costs. En The Collected Works of James M. Buchanan, vol. I, The Logical Foundations of Constitutional Liberty (pp. 260-277). Berlin: Duncler \& Humblot. También en el vol. II de la colección Medema.

Coase, R. H. (1937). The Nature of the Firm. Economica, NS 4, November. Reproducido en R. H. Coase, The Firm, the Market, and the Law (pp. 33-55), 1990. Chicago: University of Chicago Press.

Coase, R. H. (1959). The Federal Communications Commission. Journal of Law \& Economics, 2, 1-40. October.

Coase, R. H. (1960). The Problem of Social Cost. Journal of Law \& Economics, 3, 1-44. October. Reproducido en R. H. Coase, The Firm, the Market, and the Law (pp. 95156), 1990. Chicago: University of Chicago Press.

Coase, R. H. (1974). The Lighthouse in Economics. Journal of Law \& Economics, 17(2), 357-376.

Coase, R. H. (1988). The Firm, the Market, and the Law. Chicago and London: University of Chicago Press.

Coase, R. H. (1990). Notes on the Problem of Social Cost. Journal of Law \& Economics. Reproducido en R. H. Coase, The Firm, the Market, and the Law (pp. 157-185), 1988-1990. Chicago: University of Chicago Press.
Coase, R. H., \& Wang, N. (2012). How China Became Capitalist. Basingstoke: Palgrave MacMillan.

Cooter, R. (1982). The Cost of Coase. Journal of Legal Studies, 11, 1-33. January. También en S. G. Medema (Ed.), The Legacy of Ronald Coase, vol. II (pp. 92-128), 1995.

Medema, S. G. (1995). Finding His Own Way: the legacy of Ronald Coase in economic analysis. Introduction to the two-volume collection of articles on Ronald Coase, published as an Elgar Reference Collection. Aldershot.

Mill, J. S. (1848, 1965). Principles of Political Economy and Some of Their Applications to Social Philosophy. Vols. I \& II of Collected Works of John Stuart Mill. University of Toronto Press. Traducido como Principios de Economía Política. México: Fondo de Cultura Económica.

Ostrom, E. (2012). The Future of the Commons. Beyond Market Failure and Government Regulation. Indiana University.

Pigou, A. (1932). The Economics of Welfare. London: Macmillan and Co. Accessed on 24 July 2013. Library of Economics and Liberty. http://www.econlib.org/library/NPDBooks/Pigou/pgEW.html

Popper, K. R. (1959). The Logic of Scientific Discovery. London: Hutchison \& Co.

Schwartz, P. (1972). The New Political Economy of John Stuart Mill. London: George Weidenfeld \& Nicholson.

Sen, A. K. (1970). Collective Choice and Social Welfare. Edinburgh and London: Oliver \& Boyd.

Stigler, G. J. (1966). The Theory of Price. Third edition. New York: MacMillan.

Stigler, G. J. (1988). Memoirs of an Unregulated Economist. New York: Basic Books.

Voltaire, François Marie Arouet, dit Voltaire (1759). Candide ou l'optimisme. https://oll.libertyfund.org/titles/973

Williamson, O. E. (1985). The Economic Institutions of Capitalism: Firms, Markets, Relational Contracting. Free Press. 